\title{
Universiteit
}

Leiden

The Netherlands

\section{Critical scaling in linear response of frictionless granular packings near jamming}

Ellenbroek, W.G.; Somfai, E.; Hecke, M.L. van; Saarloos, W. van

\section{Citation}

Ellenbroek, W. G., Somfai, E., Hecke, M. L. van, \& Saarloos, W. van. (2006). Critical scaling in linear response of frictionless granular packings near jamming. Physical Review Letters, 97(25), 258001. doi:10.1103/PhysRevLett.97.258001

Version: $\quad$ Publisher's Version

License: $\quad$ Leiden University Non-exclusive license

Downloaded from: https://hdl.handle.net/1887/62826

Note: To cite this publication please use the final published version (if applicable). 


\title{
Critical Scaling in Linear Response of Frictionless Granular Packings near Jamming
}

\author{
Wouter G. Ellenbroek, ${ }^{1}$ Ellák Somfai, ${ }^{1, *}$ Martin van Hecke, ${ }^{2}$ and Wim van Saarloos ${ }^{1}$ \\ ${ }^{1}$ Instituut-Lorentz, Universiteit Leiden, Postbus 9506, 2300 RA Leiden, The Netherlands \\ ${ }^{2}$ Kamerlingh Onnes Lab, Leiden University, Postbus 9504, 2300 RA Leiden, The Netherlands \\ (Received 5 April 2006; revised manuscript received 6 October 2006; published 22 December 2006) \\ We study the origin of the scaling behavior in frictionless granular media above the jamming transition \\ by analyzing their linear response. The response to local forcing is non-self-averaging and fluctuates over \\ a length scale that diverges at the jamming transition. The response to global forcing becomes increasingly \\ nonaffine near the jamming transition. This is due to the proximity of floppy modes, the influence of which \\ we characterize by the local linear response. We show that the local response also governs the anomalous \\ scaling of elastic constants and contact number.
}

DOI: 10.1103/PhysRevLett.97.258001

The general picture of jamming which was advanced for systems [1-4] that form a shear-resistent solid phase at high densities is bringing a new perspective to the deformations of granular and disordered media. A good model for studying such media are packings of polydisperse weakly compressible spheres $[3,4]$. If we measure pressure in units of the elastic constants and characteristic radius of the balls (as we will do below), the relevant limit for granulates is the small-deformation or, equivalently, the small-pressure limit in the absence of thermal fluctuations. This limit is also relevant for weakly compressed emulsions [5]. We will focus on the case of frictionless, deformable spherical particles, and introduce a simple, experimentally accessible and local measure to characterize the nature of their deformations [6].

Deformable particles form a stiff jammed phase when the pressure becomes larger than zero. At the zero pressure jamming point $J$, packings form a "marginal solid" and are isostatic, i.e., the average number of contacts per particle $z$ reaches the minimum $z_{\text {iso }}^{0}=2 d$, needed for a frictionless packing to remain stable in $d$ dimensions. When the point $J$ is approached by decreasing the pressure, several surprising scaling relations emerge: the excess contact number $\Delta z=z-z_{\text {iso }}^{0}$ scales as $\sqrt{\delta}$, with $\delta$ the typical dimensionless compression of the particles, while the ratio $G / K$ of the shear modulus $G$ to the compression modulus $K$ scales as $\Delta z$. In addition, a diverging time scale $\omega^{*} \sim \Delta z$ has been identified in the density of states of vibrational modes. The jamming point $J$ thus exhibits features of a critical point [2-4].

Since packings at the jamming point are marginal, every additional broken contact generates a global zero-energy displacement mode, a so-called floppy mode [3,8-10]. Wyart and co-workers [9-11] have shown that the scaling near $J$ is related to those floppy modes, by creating trial modes for the deformations of weakly jammed solids. These modes are based on the floppy modes that would occur when along the faces of cubes of linear size $\ell^{*} \sim 1 / \Delta z$ bonds would be cut. Even though for jammed systems truly floppy modes never occur, their
PACS numbers: 45.70. $-\mathrm{n}, 05.40 .-\mathrm{a}, 46.65 .+\mathrm{g}, 64.60 .-\mathrm{i}$

proximity governs the scaling just above the jamming point.

In this Letter we uncover that this proximity of floppy modes causes an increasingly nonaffine response when approaching point $J$, and that this response is intimately related to the (anomalous) scalings of the shear modulus $G$, the excess contact number $\Delta z$, and the length scale $\ell^{*}$. We numerically study the linear, quasistatic response of systems near the jamming transition. The response of granular media has been widely studied [12-18], but not, we believe, systematically as a function of the distance to the jamming point $J$. Nor does it seem to have been fully appreciated that the scaling behavior can essentially be captured within linear response.

We represent the linear response by relative displacements and changes in contact forces, and find significant changes with the distance to point $J$. (i) Fig. 1 illustrates that the response to the loading of a single grain becomes increasingly disordered over an increasingly large scale when the jamming transition is approached, this leads to a direct observation of the diverging length scale $\ell^{*} \sim$ $1 / \Delta z$, shown below. We will show that such a local force response is not self-averaging, even though it is smooth upon ensemble-averaging and then quantitatively agrees with continuum elastic behavior. (ii) The response to a
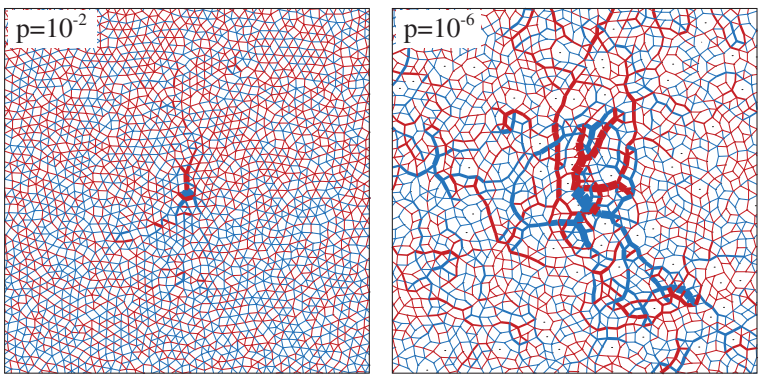

FIG. 1 (color). Force response networks for a point loading with pressure as indicated. Blue (red) lines indicate positive (negative) changes in contact force, the thickness indicating the amount. The particles themselves are not drawn. 
uniformly applied compression or shear also varies with the distance to jamming. We introduce the distribution $P(\alpha)$ of angles $\alpha$ between the bonds and the local deformations as an indicator of the nonaffine nature of the response. Near $J, P(\alpha)$ becomes strongly peaked around $\alpha=\pi / 2$, with the width and height of the peak scaling with the distance to the jamming point. Grains then predominantly slide past each other, which signals an increasingly nonaffine response of the material caused by the proximity of floppy modes, for which $P(\alpha)=\delta(\alpha-$ $\pi / 2$ ). (iii) The component of the relative displacements perpendicular to the bond vector diverges upon approaching the jamming point. (iv) Finally, the $\Delta z \sim \sqrt{\delta}$ scaling [3] is identified to originate precisely from this increasingly "sliding" response.

Hence a simple picture emerges: the influence of floppy modes can be quantified by the local linear response of the material, which becomes increasingly nonaffine near jamming, in turn causing anomalous scaling.

Linear response. - The response of a jammed granular medium to external loads has been studied mostly by full scale molecular dynamics $[14,15]$. We calculate it here in linear order from expansion of energy to second order

$$
\Delta E=\frac{1}{2} \sum_{\langle i j\rangle} k_{i j}\left(u_{\|, i j}^{2}-\frac{f_{i j}}{k_{i j} r_{i j}} u_{\perp, i j}^{2}\right) \text {. }
$$

Here the sum is over all contacts, $\vec{u}_{i}$ is the displacement of particle $i$, and $\vec{u}_{i j}=\vec{u}_{j}-\vec{u}_{i}$ the relative displacement of grains $i$ and $j$, with components $u_{\|, i j}$ and $u_{\perp, i j}$ parallel and perpendicular to the bond vector $\vec{r}_{i j}=\vec{r}_{j}-\vec{r}_{i} ; k_{i j}$ denotes the stiffness of the contact and $f_{i j}$ its initial force. The second term proportional to $u_{\perp, i j}^{2}$ is due to the transverse motion when the bonds are prestressed $\left(f_{i j} \neq 0\right)$. For contact interactions which increase as a power of the overlap $f_{i j} \sim \delta_{i j}^{\beta}$, the factor $f_{i j} / k_{i j} r_{i j}=\delta_{i j} /\left(\beta r_{i j}\right)$ is of order of the dimensionless compression $\delta=\delta_{i j} / r_{i j}$, which is small and which vanishes at the jamming point.

We study 2D packings of $N$ frictionless Hertzian spheres for which $f_{i j} \sim \delta_{i j}^{3 / 2}$, where $\delta_{i j}$ is the overlap between neighboring particles. The confining pressure ranges from $p=10^{-6}$ to $p=10^{-1}$, in units of the effective Young modulus of the constituent particles. See Ref. [19] for details. For each packing, the expansion (1) yields the dynamical matrix $M$. Instead of studying the vibrational dynamics $[7,9,10,20]$ we obtain here the quasistatic response to external forces $f^{\text {ext }}$ [21] by solving the linear equation $M_{i j, \alpha \beta} u_{j, \beta}=f_{i, \alpha}^{\text {ext }}$, for the $u_{j}$ and, through the force law, the forces $f_{i j}$. Here $i, j$ label the particles and $\alpha, \beta$ the coordinate axes.

Elastic moduli.-We have calculated the elastic moduli from the linear response by applying an overall compression or shear and by point-loading a single particle, for packings with $N=10^{3}$ and $N=10^{4}$, respectively. The resulting force fields are translated into local stress fields [18] which are then ensemble averaged. From fits of the point response [22], we determine both $K$ and $G$ and compare these to the values obtained from the response to global shear and compression. Figure 2(a) shows that these two methods agree very well quantitatively, and that the elastic moduli scale with pressure as $K \sim p^{1 / 3}, G \sim$ $p^{2 / 3}$, in agreement with earlier results [3,23].

Nonaffinity. -The typical bond stiffness $k_{i j}$ is proportional to $p^{1 / 3}$ for Hertzian contacts. Hence, a simple estimate for the elastic moduli scaling as $p^{1 / 3}$ follows under the affinity assumption that the bond deformations are of order of the applied deformation. This estimate fails for the shear modulus $G$, which vanishes faster than $K$ when approaching the jamming point: $G / K \sim \Delta z$. This has been thought to be caused by strongly nonaffine behavior of the system under shear [3] and the proximity of the floppy modes [11]. We will elucidate now the cause of the scaling and the influence of the floppy modes via the local deformations $u_{\|, i j}$ and $u_{\perp, i j}$.

As the eigenmodes or snapshots of the response look very disordered [11,19-21], it has turned out to be difficult to find a simple measure to characterize the nonaffinity and the overall floppy-mode character. We show now that proximity of the floppy modes can clearly be identified in the distribution $P(\alpha)$ of the local angles $\alpha_{i j}=$ $\operatorname{atan}\left(u_{\perp, i j} / u_{\|, i j}\right)$. In a disordered, isotropic system, one expects $P(\alpha)=\delta(\alpha-\pi)$ for a purely homogenous compression, and $P(\alpha)=1 / \pi$ for a purely affine shear. In contrast, for a floppy mode, $P(\alpha)=\delta(\alpha-\pi / 2)$. This is because in floppy modes the relative angles between particles change while the relative distances $r_{i j}$ remain unchanged, as if all the bonds are replaced by incompressible sticks [8]. Hence, for a true floppy mode $u_{\|, i j}=$ $-u_{\perp, i j}^{2} / 2 r_{i j}+\mathcal{O}\left(u_{\perp, i j}^{4} / r_{i j}^{3}\right)[24]$.
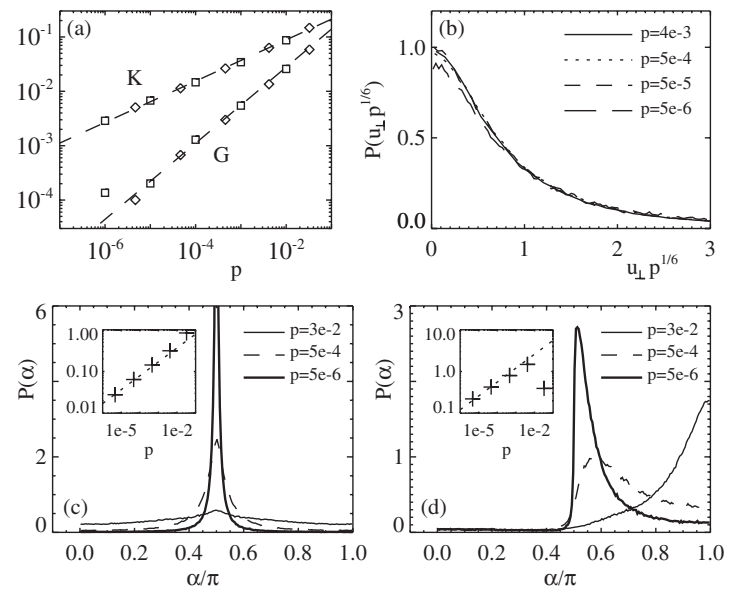

FIG. 2. (a) Scaling of bulk or shear modulus with pressure, obtained from point response (squares), and global compression or shear (diamonds). The fitted exponents are $0.38 \pm 0.03$ for the bulk and $0.70 \pm 0.08$ for the shear modulus. (b) Distribution function of the scaled transverse response for shear (see text). (c),(d) Distribution of the relative displacement angle $\alpha$ for (c) shear and (d) compression of packings for a range of pressures. Insets: scaling of the width of the peak $\sim p^{1 / 3}$. 
As the pressure is lowered, $P(\alpha)$ and $P\left(u_{\perp}\right)$ evidence that the local deformations evolve from near-affine to extremely nonaffine, floppy-modelike [Figs. 2(b)-2(d)]. Indeed, for a sheared system, $P(\alpha)$ evolves from a flat distribution at large pressures to a sharply peaked distribution for lower pressures [Fig. 2(c)]. This peak is located around $\pi / 2$ and its weight approaches 1: locally the response becomes more and more transverse for $p \rightarrow 0$ and $P(\alpha)$ approaches that of a floppy mode. For a compressed system at large pressures, $P(\alpha)$ has the "affine" peak around $\alpha=\pi$, while for lower pressures $P(\alpha)$ again develops a sharp peak around $\alpha=\pi / 2$ [Fig. 2(d)].

Even though the response is far from affine for both compression and shear, the affine prediction for $K$ holds true while it fails for $G$. The reason is that for compression, only a finite fraction of the displacements is essentially transverse and $P(\alpha)$ remains nonzero away from the peak at $\pi / 2$. Since according to the energy expression (1) the compression of bonds given by $u_{\|, i j}$ gives the dominant contribution to $\Delta E$, this is consistent with the fact that the compression modulus scales with the bond stiffness $k: K \sim$ $k \sim p^{1 / 3}$. For shear deformations, however, fewer and fewer bonds contribute to leading order to the energy, and the weight outside the peak vanishes as $\Delta z \sim p^{1 / 3}$, consistent with the scaling $G / K \sim \Delta z$.

Scaling of $P(\alpha)$ and $P\left(u_{\perp}\right)$. - We can understand the development of the peak in $P(\alpha)$ from the balance of terms in the energy expansion (1). Focussing on typical values, $\Delta E \sim k\left(u_{\|}^{2}-\delta u_{\perp}^{2}\right)$. Since $k \sim p^{1 / 3}$ and $\delta \sim \Delta z^{2} \sim p^{2 / 3}$, balancing the terms we find that

$$
\frac{u_{\|}}{u_{\perp}} \sim \sqrt{\delta} \sim p^{1 / 3},
$$

so that for small $p, P(\alpha)$ develops a peak around $\alpha=\pi / 2$, and the width of this peak should scale as $p^{1 / 3}$. This is what we find; see the insets of Figs. 2(c) and 2(d).

How do the typical values $u_{\|}$and $u_{\perp}$ scale when we impose a global shear or compression of order $\gamma$ on the system? Equating the elastic energy densities for compression and shear, $K \gamma^{2}$ and $G \gamma^{2}$, to the energy expansion, and knowing that the elastic moduli scale as $G \sim p^{2 / 3}$ and $K \sim$ $p^{1 / 3}$, we can predict the scaling of $u_{\|}$and $u_{\perp}$ :

$$
\begin{array}{cc}
\text { Compression : } u_{\|} \sim p^{0} \gamma, \quad u_{\perp} \sim p^{-1 / 3} \gamma \\
\text { Shear: } u_{\|} \sim p^{1 / 6} \gamma, \quad u_{\perp} \sim p^{-1 / 6} \gamma
\end{array}
$$

These scaling predictions are well obeyed by our data for small $p$ : in Fig. 2(b) we show this for shear deformations and $P\left(u_{\perp}\right)$.

The fact that for fixed $\gamma$ the typical perpendicular response $u_{\perp}$ diverges upon approaching the jamming point, is connected to the disordered nature of the microscopic response already familiar from the randomly oriented swirl-type motions $[11,19,20]$ that characterize eigenmodes and responses. Of course, in a system of finite size, $u_{\perp}$ cannot diverge. If the crossover is determined by the length scale $\ell^{*}$ becoming of order the linear system size $L$, one expects a crossover scaling $u_{\perp}=L^{1 / 2} g\left(\ell^{*} / L\right)$ with $g(w) \sim w^{1 / 2}$ for $w \ll 1$ and $g \simeq$ const for $w \gg 1$. Note also that in the regime $L / \ell^{*} \ll 1$ the response is close to that of a floppy mode appearing at isostaticity, while our scaling results apply to the regime $L / \ell^{*} \gg 1$.

$\Delta z$ scaling. - The nonaffine response also nicely explains the microscopic origin of the anomalous $\Delta z \sim \sqrt{\delta}$ scaling under compression: theories assuming affine deformations give $\Delta z \sim \delta$. Let us consider a small compression of the packing with typical bond compression $u_{\|}$. This leads to an infinitesimal change in contact number

$$
\frac{d \Delta z}{d \delta} u_{\|}
$$

Upon lowering the pressure, the global compression will excite distorted floppy modes; i.e., for many bonds $u_{\perp, i j}$ will be of order $u_{\|} / \sqrt{\delta}$ [see Eq. (2) and Fig. 2(d)]. Moreover, the chance that in such almost perpendicular displacements one of the particles bumps into a nearby particle with which it was not in contact yet, will be proportional to this motion, i.e., to $u_{\|} / \sqrt{\delta}$. Equating this to (5) then yields $\Delta z \sim \sqrt{\delta}$. The picture which emerges is that the $u_{\perp, i j}$ and hence the chances that new contacts are created are larger at lower pressure.

In earlier papers, it was noted $[3,25,26]$ that the $\Delta z \sim$ $\sqrt{\delta}$ scaling with compression was consistent with a square root divergent term in the correlation function $g(r)$, if it was assumed that compression would be essentially affine. As we have seen, however, distortions are not at all affine near jamming. Our analysis turns this around: it suggests that the natural coordinates for the floppy-mode-like distortions are the perpendicular displacements, not the radial ones, and that these generate the square root behavior of $g(r)$ in the radial direction.

Point response and diverging length scale.-We finally return to the point response. Figure 3(a) illustrates that the ensemble average of such a response conforms to elasticity; the stress fields fit well and the fitted elastic constants agree with those obtained from bulk response [Fig. 2(a)]. On the other hand, individual responses become very disordered and suggest the occurrence of a large length scale $\ell^{*}$ when approaching point $J$ (see Fig. 1).

To extract this length scale, we characterize the response to an infinitesimal inflation of a single central grain, since the response to a local directional force, as shown in Figs. 1 and 3(a), is highly anisotropic. We normalize the forces by fitting the radial stress to the elastic response. We focus on the radial component of the change in contact force $d f_{r}$, calculate $\left\langle d f_{r}(r)\right\rangle$ by averaging $d f_{r}$ over concentric rings, and study the rms fluctuations $h(r) \equiv$ $\sqrt{\left\langle\left[d f_{r}(r)-\left\langle d f_{r}(r)\right\rangle\right]^{2}\right\rangle}$.

The range over which these fluctuations are felt grows when approaching the jamming transition [Fig. 3(b)]. When plotted as function of $r \Delta z$, the data for $h$ collapses 
(a)

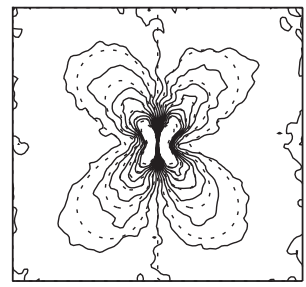

(c)

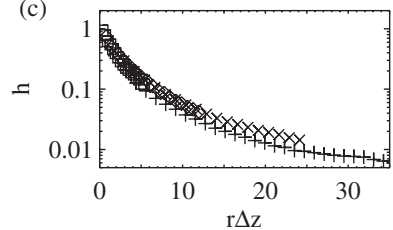

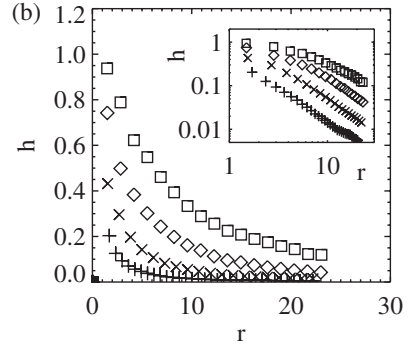

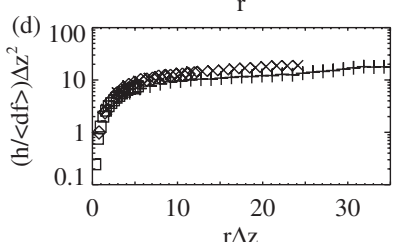

FIG. 3. (a) Shear stress $\sigma_{x y}$ for point loading in the center and $p=10^{-3}$. The response is coarse grained over a grain diameter [18] and averaged over 216 realizations (solid) and fitted to linear elasticity (dashed). (b)-(d) Spatial decay of the force fluctuations $h$. Squares, diamonds, crosses, and plus signs represent increasing pressures, ranging from $p=4.6 \times 10^{-5}$ to $p=3.2 \times 10^{-2}$, respectively. (b) $h$ as function of $r$. (c) Scaling collapse when $h$ is plotted as function of $r \Delta z$. (d) Scaling collapse of relative fluctuations.

[Fig. 3(c)]. To our knowledge, this is the first evidence for the existence of a length scale $\ell^{*} \sim 1 / \Delta z$ in local response measurements. Essentially the same characteristic length was shown by Wyart et al. to govern the vibrational density of states $[9,10]$. This scale is identified as the linear size $\ell^{*}$ of the largest domain which deforms freely by pushing on the bonds at its surface. Equating the number of bonds on the surface $\left(\sim \ell^{d-1}\right)$ to the number of excess bonds in the bulk $\left(\sim \Delta z \ell^{d}\right)$ yields the maximum size of such domain $\ell^{*} \sim 1 / \Delta z[9,10]$.

Both the average response $\left\langle d f_{r}(r)\right\rangle$ and the fluctuations $h(r)$ decay as $1 / r^{2}$; the relative fluctuations do not decay far from the perturbed grain. The response is not selfaveraging, and there is no finite correlation length of fluctuations. The asymptotic value of the relative fluctuations $h(r) /\left\langle d f_{r}(r)\right\rangle=h(r) r^{2}$ grows as $1 / \Delta z^{2}$ [Fig. 3(d)]; one has to coarse grain the response over increasingly more grains $\mathcal{O}\left((\Delta z)^{-4}\right)$ to start to see convergence to average continuumlike stress response.

Outlook. - Our analysis of the (local) linear response substantiates and extends the concept that the jammed phase of weakly compressed frictionless particles is dominated by the proximity of floppy modes [8-11]. We identified the increasingly nonaffine response to give rise to the scaling $\Delta z \sim \sqrt{\delta}$ and presented a direct observation of the scale $\ell^{*} \sim 1 / \Delta z$ introduced before $[9,10]$. The emerging scenario favors a microscopic, geometric interpretation of these scalings and has several implications that deserve further study: (i) What is the finite size scaling form of $u_{\perp}$ ? (ii) What happens for non-power-law contact interactions such as $f_{i j} \sim \exp \left(-\delta_{i j}^{(1-\beta)}\right)$ with $\beta>1$ ? Our analysis sug- gests a scaling $\Delta z \sim \delta^{\beta / 2}$. (iii) What happens to the square root divergence in $g(r)[3,25,26]$ when the packing algorithm does not allow for floppy-mode-like rearrangements upon annealing, such as may occur for algorithms based on local rearrangements or packings of truly hard spheres?

We thank K. Shundyak, J.H. Snoeijer, and M. Depken for discussions and M. Wyart for critical correspondence which led us to realize the divergence (3) and (4). W. G. E. acknowledges support from physics foundation FOM, and M. v. H. acknowledges support from NWO/VIDI.

*Present address: The Rudolf Peierls Centre for Theoretical Physics, 1 Keble Road, Oxford, OX1 3NP, U.K.

[1] A. J. Liu and S. R. Nagel, Nature (London) 396, 21 (1998).

[2] H. M. Jaeger, Phys. World 18, No. 12, 34 (2005).

[3] C.S. O'Hern et al., Phys. Rev. E 68, 011306 (2003).

[4] C.S. O'Hern et al., Phys. Rev. Lett. 86, 111 (2001).

[5] T. G. Mason, J. Bibette, and D. A. Weitz, Phys. Rev. Lett. 75, 2051 (1995).

[6] For frictional packings, the situation is more complicated: frictional packings are not automatically marginal solids at jamming, but a critical-like scaling does emerge in the large-friction limit; see [7].

[7] E. Somfai et al., cond-mat/0510506 [Phys. Rev. E (to be published)].

[8] S. Alexander, Phys. Rep. 296, 65 (1998).

[9] M. Wyart, S. R. Nagel, and T. A. Witten, Europhys. Lett. 72, 486 (2005).

[10] M. Wyart et al., Phys. Rev. E 72, 051306 (2005).

[11] M. Wyart, Ann. Phys. (Paris) 30, 1 (2005).

[12] J. Geng et al., Phys. Rev. Lett. 87, 035506 (2001); Physica (Amsterdam) 182D, 274 (2003).

[13] D. Serero et al., Eur. Phys. J. E 6, 169 (2001).

[14] A. Kasahara and H. Nakanishi, Phys. Rev. E 70, 051309 (2004).

[15] C. Goldenberg and I. Goldhirsch, Nature (London) 435, 188 (2005).

[16] S. Ostojic and D. Panja, Phys. Rev. Lett. 97, 208001 (2006).

[17] N. Gland, P. Wang, and H. A. Makse, Eur. Phys. J. E 20, 179 (2006).

[18] I. Goldhirsch and C. Goldenberg, Eur. Phys. J. E 9, 245 (2002).

[19] E. Somfai et al., Phys. Rev. E 72, 021301 (2005).

[20] A. Tanguy et al., Phys. Rev. B 66, 174205 (2002).

[21] F. Leonforte et al., Phys. Rev. B 70, 014203 (2004).

[22] W. G. Ellenbroek et al., in Powders and Grains 2005, edited by R. García-Rojo et al. (A. A. Balkema, Rotterdam, 2005), p. 377; (to be published).

[23] H. A. Makse et al., Phys. Rev. Lett. 83, 5070 (1999); Phys. Rev. E 70, 061302 (2004).

[24] For a floppy-mode distortion, the second term in (1) cancels the linear term $-\sum_{i j} f_{i j} u_{\|, i j}$ in the energy expansion. In (1) this term has been left out on account of force balance in the starting state.

[25] L. E. Silbert et al., Phys. Rev. E 65, 031304 (2002).

[26] L. E. Silbert, A. J. Liu, and S. R. Nagel, Phys. Rev. E 73, 041304 (2006). 